\title{
Comparative Study of Illumination Pre-processing Techniques using Histogram Equalization and its Application in Face Recognition
}

\author{
R. P. Dahake ${ }^{1}$, M. U. Kharat $^{2}$ and S.V. Gumaste ${ }^{3}$ \\ ${ }_{1,2,3}$ MET's Institute of Engineering, Nasik, India
}

\section{ABSTRACT}

In the last decades, automatic face recognition has become more prominent in the wide range of applications including security, judicial investigations and law enforcement etc. For practical face recognition systems different challenges are lighting, posing, expression, ageing, occlusion etc. Changing the appearance of faces is due to various lighting conditions such as shadows and exposing the light on different parts of the face. Balanced illumination in face image helps to enhance its quality which is an important parameter to increase the rate of face recognition. A better quality image provides a better detection and recognition rate than poorly illuminated images. Extraction of features for face recognition is even more difficult with such images resulting in reduction of face recognition rate hence for overcoming this problem, illumination pre-processing techniques usually adopted before face detection and extraction of features from an image. In this paper the objective is to analyse performance of contrast enhancement techniques such as Histogram Equalization, Contrast Limited Adaptive Histogram Equalization and Contrast Limited Adaptive Histogram Equalization (CLAHE) with image entropy techniques for pre-processing of poor illuminated facial images. Further two basic techniques of feature extraction such as Principal Component Analysis and Local Binary Pattern and their hybrid approach for global and local feature extraction from images are utilized for evaluation on facial images. Yale B and extended Yale B datasets are used for experimentation. CLAHE with image entropy illumination pre-processing approach outperforms as compared to Histogram Equalization and CLAHE techniques. The very first noting is, initially face detection accuracy which is poor after applying illumination pre-processing improved, detection rate $81.66 \%$ is observed. Secondly using hybrid features of facial images and CLAHE with image entropy for illumination pre-processing has shown better recognition performance as 92\% on cropped frontal faces of the same dataset.

KEY WORDS: ILLUMINATION, HISTOGRAM EQUALIZATION, PRE-PROCESSING, ENTROPY, FACE DETECTION, RECOGNITION.

\section{INTRODUCTION}

In the recent decades Automatic Face Recognition (AFR) has drawn a lot of attention because of the wide

\section{ARTICLE INFORMATION}

*Corresponding Author: dahakeranjana@gmail.com Received 18th Oct 2020 Accepted after revision 29th Dec 2020 Print ISSN: 0974-6455 Online ISSN: 2321-4007 CODEN: BBRCBA

Thomson Reuters ISI Web of Science Clarivate Analytics USA and Crossref Indexed Journal

\section{Clarivate
Analytics}

NAAS Journal Score 2020 (4.31)

A Society of Science and Nature Publication,

Bhopal India 2020. All rights reserved.

Online Contents Available at: http//www.bbrc.in/

Doi: http://dx.doi.org/10.21786/bbrc/13.14/91 applications ranging in the vast areas like security, criminological assessment, and law implementation. The framework of the AFR system needs to face difficulties like changing facial appearance, varying lighting conditions, posture, facial expression etc. Changing lighting conditions for example shadows, over and under exposure of light on face pictures are vital issues that a practical face recognition system has to address. In fact light variations cause significant changes in the appearance of face image due that intra-personal differences can be larger than inter-personal differences (Y. Adini et al., 1997). 
Although various approaches have been proposed in the past decades for dealing with light variation's effect on the face. Illumination pre-processing is a very crucial step used in recognition of faces (Hu Han et al., 2013). Most of the works addressed the illumination problem in the field of AFR. A good survey is given in (X. Zou et al., 2007). Compared to the traditional face recognition method, illumination pre-processing is more popular in face recognition, because of its simplicity and feasibility. After applying illumination pre-processing, the output still provides a face image and various feature descriptors such as Local Binary Pattern (LBP) (Jiwen Lu et al., 2015; Di Huang et al., 2011) which can be applied after illumination pre-processing. There are a number of applications of face detection and recognition. Human Computer Interaction is an important domain nowadays. Human faces are the key element to interact with the smart system as well as hand gestures are also mostly used in such systems where accuracy and efficiency plays vital roles (R M Kagalkar et al., 2015).

Though the efficiency and accuracy of face recognition are important factors that have been reduced due to facial illumination variations. Traditional methods make it difficult to find a face if it has images with heavy shadows and over-exposure. For example Viola-Jones face locator (Paul Viola et al., 2004) fails under extreme light varieties. In these conditions, face identification strategies dependent on skin discovery procedures (S. Shan et al., 2003; B. Martinkauppi et al., 2003; Leyuan Liu et al., 2011; R.P Dahake et al., 2018) may be utilized to locate the facial skin zone.

Histogram Equalization (HE) based methods are simple and effective to use in image illumination pre-processing. Low contrast images visibility can be clearly enhanced with this technique as in (N.SiaPikKong et al., 2013) a good literature review is given and some of the extensions of HE can be classified into four groups. They are Mean Brightness Preserving HE (Kim Y.-T et al., 1997), Bin Modified HE \& Local HE (S.M. Pizer et al., 1987). In Mean Brightness Preserving HE the idea of keeping the mean brightness of an image for consumer electronic product where as in Bin Modified HE modifies the shape of the image histogram by reducing or increasing the value in the histogram's bins based on a threshold limit before the equalization is taking place.

HE modifies the histogram for distributing the narrow range of gray scale values across the entire available range (Ranjan Parekh., 2006). In local HE multiple regions of image are considered. One of the variation of Local HE is Contrast Limited Adaptive Histogram Equalization. There are some approaches based on CLAHE which work on a limited number of images for deciding the clipping and other parameters using entropy curve (Byong Seok Min et al., 2013) towards enhancing image contrast. Authors in (G. F. C. Campos et al., 2019) have provided a learning-based hyper parameters selection technique for the CLAHE. In this paper, a comparative study on $\mathrm{HE}$, basic CLAHE and CLAHE with image entropy is done, further analyzing impact of illumination pre- processing in the application of face recognition. There are two important parameters of CLAHE clip limit (CL) and block size (BS) which play an important role for contrast enhancement. The strategy for deciding these two parameters with a concept CLAHE with entropy of image is adopted.

For the determination of face matching algorithm basic techniques like Principal Component Analysis (PCA) (M. Turk et al., 1991; Chris Solomon et al., 2010) and Local Binary Pattern (LBP) (Di Huang et al., 2011) are largely used facial feature extraction, one of the best in class strategies (W. Zhao et al., 2003) are selected. Moreover, two public face database Yale B (A. S. Georghiades et al., 2001) and extended Yale B (K.C. Lee et al., 2005) for analyzing impact of illumination in face recognition are utilized. Comparative analysis of face recognition methods is done on these dataset. The paper is divided as Introduction in section 1, Importance of Readings and Observations in section 2. Section 3 provides details of adopted pre-processing approaches for contrast enhancement in face recognition. In section 4 experimental strategy is given. Section 5 provides result analysis and discussions. Section 6 contains conclusions followed by references.

2. Importance of Readings And Observations: There are countless illumination pre-processing techniques in the literature. Illumination pre-processing approaches are assembled into three classes: Gray-Level Transformation, Gradient and Edge Extraction, and Face Reflection Field Estimation (Hu Han et al., 2013). These methods are elaborated in section 2.1, 2.2 and 2.3. Followed by some of the important observations of recent work under these categories.

2.1 Gray-Level Transformation: In this method the guidelines for pre-processing is to play out a pixelwise intensity planning to map using one particular transformation function. After this, the distribution of intensities in a face picture is carried out so that the unbalanced illumination can likewise be adjusted. Linearly or non-linearly the transformation functions are utilized. The non-linear transformation is more viable, the methods like Logarithmic Transform (Y. Adini et al., 1997), Histogram Equalization (HE) and Gamma Intensity Correction (S.Anila et al., 2012) can be viewed as methods in non- linear category of Gray-Level Transformation.

2.2 Gradient or Edge Extraction: This method is based on extraction of gradients or edges for illumination pre-processing on the face. Brightening pre-processing considers gray-level gradients or edges and boundaries of objects which plays a very essential role for identification. Approaches like Directional Gray-scale Derivative and Laplacian of Gaussian (Y. Adini et al., 1997) are under this category and they are dependent on gradient and edge highlights.

2.3 Reflectance Field Estimation: In this method brightening pre-processing is to gauge the face reflectance field from a two dimensional face picture. 
Clearly, the face reflectance field is light invariant. For example a reflectance-brightening model which speaks to a face picture as the result of face reflectance and light segment. Some of approaches under this category are Logarithmic Total Variation and Self Quotient Image (SQI).

Numerous pre-preparing techniques work absolutely to determine all around controlled light varieties, yet acknowledgment in uncontrolled circumstances is one of the most significant bottlenecks for viable face acknowledgment frameworks (X. Tan et al., 2010). In (W. Chen et al., 2006) Discrete Cosine Transform is employed to compensate for illumination variations in the logarithm domain space. Authors in (J. Ruiz-del-Solar et al., 2008) have worked on the Self- Quotient Image and demonstrated that the most appropriate techniques for achieving light compensation and normalization using eigenspace for recognition of faces are (SQI) and the modified LBP transform. Whereas authors in (X. Xie et al., 2008) a single face image is first decomposed into large -and small -scale feature images using logarithmic total variation.

Ping-Han et al. proposed the Orientated Local Histogram Equalization technique. This technique uses the edge orientations for compensation of illumination (Ping-Han Lee et al., 2012). Regardless of the accomplishment of these algorithms, as per analysis of authors in (H. F. Chen et al., 2000) there are no discriminative functions of images of objects that are in variant to illumination.

Histogram Equalization (HE) is many times valuable for contrast enhancement, only the thing is, it functions admirably just when the face picture is darkened globally. CLAHE and Local Histogram Equalization (LHE) (S. Pizer et al., 1990) are more powerful in repaying local lighting varieties. CLAHE is proposed by the author in ( K. Zuierveld 1994), its two important parameters are block size and clip limit. These parameters are basically used for controlling quality of image, but the user determines them heuristically. CLAHE was originally developed for medical imaging (S.M. Pizer et al 1987). One of the modified CLAHE approach for auto defining clip limit is (Jharna Majumdar et al., 2014) where authors have worked on enhancement of aerial, medical and underwater images using modified CLAHE techniques. Method of image enhancement with brightness preserving histogram equalization with maximum entropy is given in (C. Wang et al., 2005).

An approach using enhancement in CLAHE technique is proposed to provide a face recognition system that is useful for invariants of illumination. Block Size 8X8 \& clip limit 0.01 are applied and testing of work on simple and elementary image dataset is done (A. Thamizharasi et al., 2016). In the CLAHE method for getting satisfactory results the correct block size and clip limit need to be determined. Work in the paper is towards this direction with evaluation of low contrast facial images.
3. Contrast Enhancement For Face Recognition: Illumination pre-processing is one of the essential steps in face recognition frameworks as shown in Fig. 1. Face recognition block contains a series of steps. One of the important steps is detection of faces. If the input images are provided with very low contrast and visually dark as shown in Fig. 2 then illumination pre-processing is must for successful face detection otherwise lighting variation also affects detection performance. In most of work illumination processing is carried before the feature matching phase in the framework, but in this work it is also applied before detection of faces with the novel thinking that lighting variations impact on faces. It degrades not only the face recognition performance but it also reduces the face detection accuracy. It is the initial step in a face recognition block.

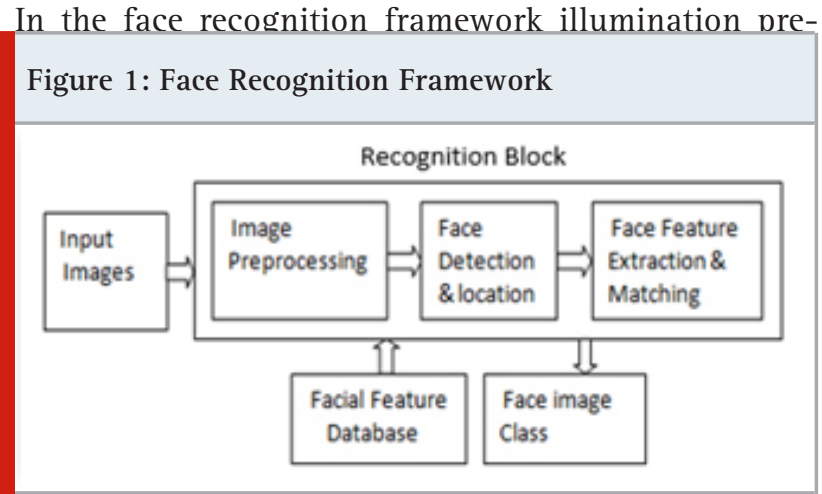

processing applied before detection and feature matching phase. Three illumination pre-processing techniques independently processed namely HE and basic CLAHE and CLAHE with image entropy. When CLAHE with image entropy pre-processing used then initially finding the range of entropy values of every image in the dataset, the second step is applying CLAHE. However, this method relies on two essential parameters: the block size and the clip limit which are defined in further sections. For face detection Viola Join face detector is used. For feature extraction selected techniques are PCA and LBP as well as hybrid features of combining these two methods. A holistic approach is used for the entire face area and Principal Component Analysis is to represent feature space.

Local features of facial components like eyes features are extracted from face for performing hybrid approach. Both local and global features are considered just like a human identity system uses both local features and full face regions for recognizing a face, the machine recognition system also uses both features. Input images are from more challenging facial dataset Yale B and extended Yale B containing a huge set of images with a large range of contrast and intensity variations. These set of images are input to the face recognition block. Matching between facial features database images and testing probes using Euclidean distance measure. Finding the face image class is recognizing the specific subject image from the dataset. Further in section 3.1 exploring the entropy of image, image histogram. Illumination 
pre-processing approaches for enhancing the contrast of dark facial images namely HE, CLAHE and CLAHE with image entropy are explained in following sections 3.2, 3.3, 3.4.

3.1 Image Entropy: The shape of the gray level histogram can provide an idea about the overall appearance of the image. For an illustrative face from extended Yale B dataset and corresponding histogram of the face image is shown in image Fig 2. The first two are the actual face images with their relevant histograms i.e. (a), (b) and (c) is the cropped face from the corresponding image. An image with a positive-skew gray level histogram looks brighter than with a negative-skew gray level histogram because the total pixels with higher gray values in the previous image is greater than the latter image. If there are $\mathrm{L}$ gray levels with probability $\mathrm{p}_{\mathrm{i}}$ associated with $\mathrm{i}^{\text {th }}$ event $(\mathrm{i}=0,1,2 \ldots, \mathrm{L}-1)$ such that $\sum_{-}(\mathrm{i}=0)^{\wedge}(\mathrm{L}-1 \quad) \sum_{i=0}^{t=1} p_{i}=1$, then the entropy or information content can be defined in eq. (1).

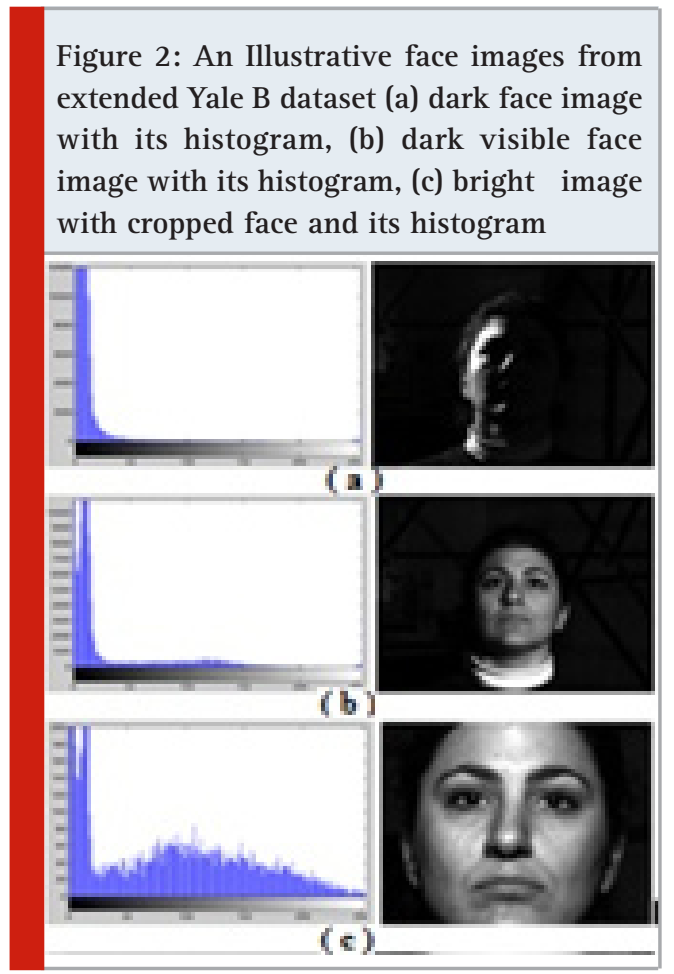

Entropy of grayscale image I is represented by E, a scalar value. It is a statistical measure of randomness and used to characterize the input image texture value (R. Gray., 1990, C. Wang et al., 2005, Byong Seok Min et al., 2013).

$$
E=-\sum_{i=0}^{L-1} p\left(x_{i}\right) \log p\left(x_{i}\right)
$$

Here $p\left(x_{i}\right)$ is the normalized probability of the gray level $\mathrm{xi}$. The entropy is maximal when $\mathrm{p}\left(\mathrm{x}_{\mathrm{i}}\right)$ is constant. When the incident frequency is the same across all gray levels, entropy is increased in the image. That is, the gray level histogram will be uniform. The Gray level histogram reveals the overall appearance of the image (B. Chanda et al., 2009).

3.2 Histogram Equalization: The main idea of $\mathrm{HE}$ is to redistribute the gray-level values uniformly (M.Anji Reddy et al., 2009). The contrast of the grayscale image indicates how easily objects in the image are recognizable. High intensity images have several different intensity values where low contrast images have low intensity values. In Histogram Equalization, contrast enhancement is proportional to the histogram's height at that intensity. Cumulative Density Function (CDF) is calculated by eq. (3). For making a uniform intensity distribution HE re-maps the gray levels of input image by re-assigning intensity values of pixels (Rafael Gonzalez et al., 2009; Anil K. Jain., 2008). The process of histogram equalization is as follows (Kim Y.-T., 1997)

Image $I=\{I(i, j)\}$ and denoting the $\mathrm{L}$ discrete gray levels as $\left\{\mathrm{I}_{0}, \mathrm{I}_{1} \ldots . . \mathrm{I}_{\mathrm{L}-1}\right\}$, an intensity of $\left.\mathrm{I}_{\mathrm{i}, \mathrm{j}}\right)$ at the spatial location $(\mathrm{i}, \mathrm{j})$ and $\left.\mathrm{I}_{\mathrm{i}, \mathrm{j}}^{\mathrm{L}}\right) \varepsilon\left\{\mathrm{IO}, \mathrm{I}_{1} \ldots . . \mathrm{I}_{\mathrm{L}-1}\right\}$ and $\mathrm{p}(\mathrm{Ik})$ is Probability Density Function(PDF) as defined in eq. (2).

$$
p\left(I_{k}\right)=\frac{n^{k}}{n}
$$

for $\mathrm{k}=0,1, \ldots, \mathrm{L}-1$, where $\mathrm{n}^{\mathrm{k}}$ represents the number of times $I_{k}$ appears in the given image $I$ and $n$ is the total number of samples in the image. $I_{k}$ associates with histogram of a given image which represents the number of pixels with intensity $I_{k}$. A plot of $n^{k}$ vs. Ik is referred to as histogram of $\mathrm{I}$. C $(\mathrm{x})$ is defined on the basis of PDF.

$$
C(x)=\sum_{j=0}^{k} p\left(I_{j}\right)
$$

Where $\mathrm{X}_{\mathrm{k}}=\mathrm{x}$, for $\mathrm{k}=0,1, \ldots, \mathrm{L}-1$ and $\mathrm{C}\left(\mathrm{X}_{\mathrm{L}-1}\right)=1$ by definition. As HE maps input image into the entire dynamic range, $\left(\mathrm{I}_{0}, \mathrm{I}_{\mathrm{L}-1}\right)$ by using $\mathrm{CDF}$ as a transform function as defined in eq. (3). HE is based on the whole information of the input image to implement, the local details with smaller probability would be less enhanced.

\section{Contrast Limited Adaptive Histogram Equalization(CLAHE):}

CLAHE is another technique for contrast enhancement for poorly illuminated images. It is one of the Adaptive Histogram Equalization approaches. Several histograms are called sub histograms, for emphasizing local contrast, instead of overall contrast. Fig. 3 represents basic steps of CLAHE using a sample image. CLAHE divides the images into equal regions. These regions are also called tiles. Number of tiles are specified by block size. Histogram of every region is generated by eq. (2) further it applies the histogram equalization to each region. The histogram is clipped to a predetermined value before calculating CDF described by eq. (3) and therefore limits the CLAHE amplification. CLAHE overcame the limitations of standard histogram equalization. The two key parameters: block size and clip limit are mainly used to control image quality which has been heuristically determined by users. 
Figure 3: Process of Contrast Limiting Adaptive Histogram Equalization.

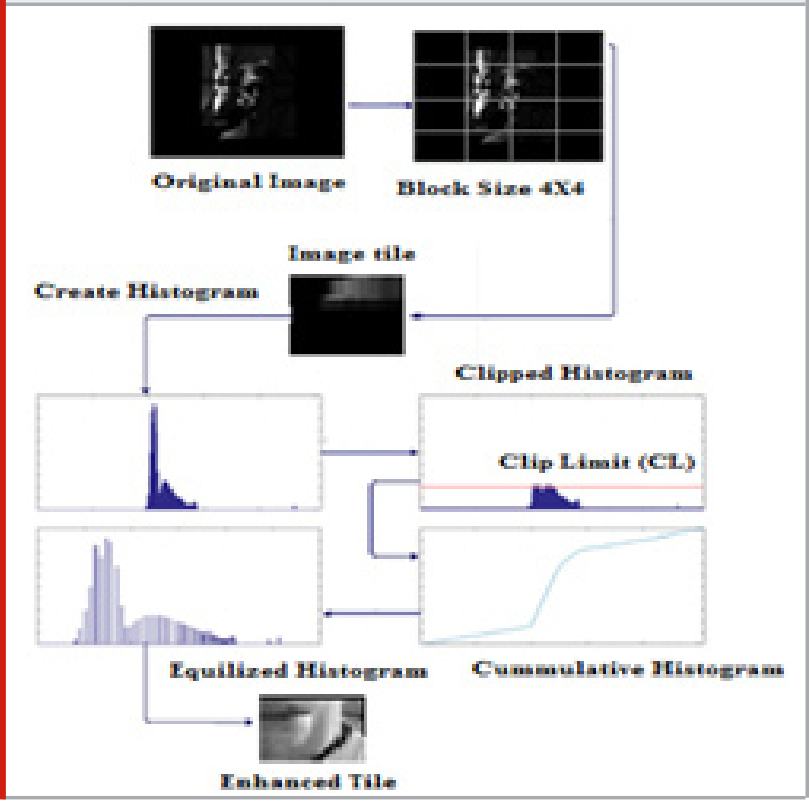

Algorithm 1 is basic CLAHE, Clip limit is the histogram clipped value and it depends on the generalization of the histogram and the size of the neighboring area. This performs uniform distribution of grey values which helps for providing a more visible image with hidden features. The full grey spectrum is utilized for expressing the image.

\section{Algorithm 1: Basic CLAHE}

begin

Input: face image

Output: image with improved contrast

(Parameters: $\mathrm{CL}=0.01, \mathrm{BS} 8 \mathrm{x} 8$ )

1. for all tiles of an image

2. Extract single image region

3. Perform Histogram equalization on each tile using a pre-defined clip limit.

4. Interpolating gray level effects at the join boundaries of region to assemble the final image

5. Image with improved contrast end

CLAHE works on tiles, instead of the whole face picture. It calculates the contrast transform function for each tile independently. Each tile's contrast is improved, so the histogram of the output region approximately matches the histogram determined by the distribution value which is considered as uniform. CLAHE other parameters such as distribution type, bin size etc. are need to set for the same. The neighboring tiles are then joined utilizing bilinear interpolation to eliminate artificially induced boundaries. The contrast, particularly in homogeneous regions, can be restricted to avoid intensifying any noise that may be available in the face picture. The ultimate result of CLAHE depends on the CL value picked by the user, if default values are used then for varied low contrast input images may not be adaptively adjusted.

3.4 Contrast Limited Adaptive Histogram Equalization with Image Entropy: Clip limit is a contrast factor that prevents oversaturation of the image specifically in homogeneous areas. These areas are characterized by a high peak in the histogram of the particular image tile due to many pixels falling inside the same gray level range. In basic CLAHE pre-processing approach the values of two parameters clip limit and block size are heuristically selected by user for avoiding this trial basis input of these parameters, in CLAHE with entropy pre-processing for contrast enhancement is adapted. Algorithm 2 provides details for determining clip limit for the CLAHE technique. Image entropy calculated as per described in eq. (1). Clip limit is decided from the entropy value which is considered a quality parameter for a given image. Initial CL value can be incremental with step value given. Clip limit is determined at the saturation point of the entropy value of the image. As shown in Fig. 4 in the plot of clip limit verses entropy value of image, for the given value of block size as increases clip limit, with that, the value of image entropy after some initial values it remains constant for further change in the value of clip limit at this saturated point CL is determined.

\section{Algorithm 2: CLAHE with Image Entropy}

begin

Input image file

Output: image with improved contrast

Initialize all input parameters of basic CLAHE

$\mathrm{CL}=0.01, \mathrm{BS}=4 \times 4$,

Array of entropy values: entropy,

Step value: incremental value

1. Read image

2. Calculate image entropy by eq. (1)

3. Store entropy value

4. Increment value of CL by stepvalue

5. Repeat step 2 and 4 till entropy value remains constant (Comparing previous one value)

6. Sort all the entropy value and CL values

7. Select CL at maximum value of entropy

8. Process CLAHE on image

9. Repeat step 1 to 8 for all images

10. end

4. Experimental Strategy: Following sections (4.1, 4.2 and 4.3) provides dataset detail, process of experimentation and result analysis and discussion.

4.1 Dataset Details: For evaluation of the illumination pre-processing approaches discussed in this paper Yale B and extended Yale B datasets are used. In Yale B dataset a total 10 individual facial images are provided, each individual's images are captured under 64 various lighting conditions. Total 9 pose or viewing angles are there with frontal face as pose 0. Extended Yale B consists of 16128 face images. There are 28 different 
individual's facial images. These images were captured under the same illumination conditions as the images in Yale B. For further evaluation a combined Yale B and extended Yale B data set is formed. It contains faces of 38 individuals.

4.2 Process of Experimentation: Experimentation is carried out in two phases initially pre-processing techniques HE, Basic CLAHE with fixed parameters $\mathrm{CL}=0.01$ and $\mathrm{BS}=8 \mathrm{X} 8$ and CLAHE with entropy applied independently then the evaluation process for face detection and recognition of facial images is carried out in second phase. Setup for implementation: Matlab on 64 bit operating system with Intel(R)Core(TM) i7-7500U CPU@2.70GHZ and RAM: 8.00 GB.

In table 1 two important parameters details are listed used for pre-processing for CLAHE with image. Block size is a two-element vector. It's a positive integer specifying the number of tiles by row $\&$ column. Product of row and column is consider as the total number of tiles. A clip limit is real scalar in the range [0 1] in Matlab and others. That specifies a contrast enhancement limit.

Table 1. Key Parameters Range of CLAHE with Image Entropy

\begin{tabular}{|l|l|c|c|}
\hline Symbol & Parameter & Range & Step \\
\hline CL & Clip Limit & {$[0, \ldots, 1]$} & 0.01 \\
\hline BS & Block Size & {$[4, \ldots, 32]$} & 2 \\
\hline
\end{tabular}

Figure 4: Image Entropy with varying clip limit

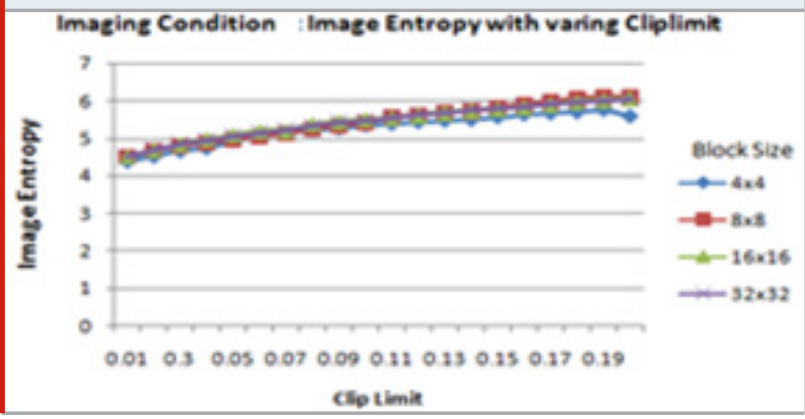

4.3 Experimental Results: In the selected dataset there are 64 varying illumination conditions for each pose so we observed entropy values for various imaging illumination conditions as well as the entropy value with all possible values of clip limit up to the saturation level. Illustrative graphical representation is shown in Fig. 4. Initially the value of $\mathrm{BS}$ is kept constant at $\mathrm{BS}=4 \times 4$ Ct CL value is varied to 1 Then the experimentation is repeated for the value of $\mathrm{BS}=4 \times 4,8 \times 8,16 \times 16$ and $32 \times 32$. A saturated point is shown for deciding the clip limit for this approach. An exemplary image with a particular illumination condition which is having very low entropy values and thus the image under such illumination conditions are more difficult to detect and recognizes. Thus for face detection and recognition appropriate CL value is to determine on the basis of entropy values of that image.

Darker images have less visibility as shown in Fig. 5 (a). 5 (b) is a face image after Histogram Equalization. This image is visible but due to over amplification some local details are lost, 5(c) basic CLAHE preprocessed image is shown with fixed parameters values $\mathrm{CL}=0.01$ and $\mathrm{BS}=$ 8x8. From Fig. 5(d) to 5(h) CLAHE with image entropy for illumination pre-processing with changing values of CL 0.01 to 0.1 and fixed $B S=4 \times 4$ on same the input image showing enhancement in visual appearances.

Table 2. Varying illumination and pose face detection performance on extended Yale B dataset

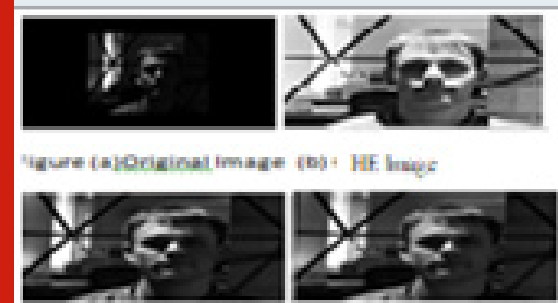

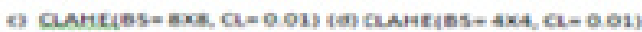

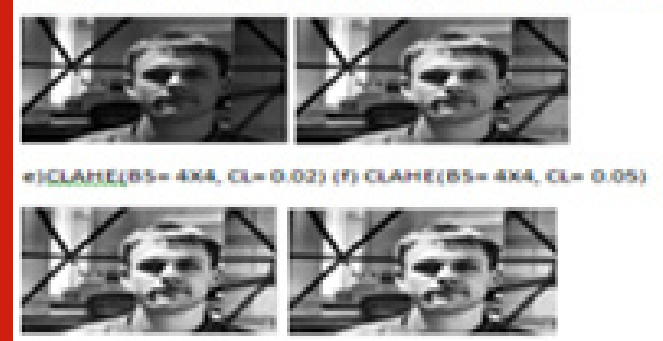

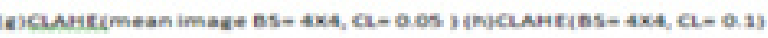

Table 2. Varying illumination and pose face detection performance on extended Yale B dataset

\begin{tabular}{|l|c|c|}
$\begin{array}{l}\text { Image } \\
\text { Set }\end{array}$ & $\begin{array}{c}\text { Method of Illumination } \\
\text { Pre-processing }\end{array}$ & $\begin{array}{c}\text { Detection } \\
\text { Accuracy (\%) }\end{array}$ \\
\hline \multirow{2}{*}{ Set0 } & Before Pre-processing & 67 \\
& Histogram Equalization & 91 \\
& CLAHE With image Entropy & 91 \\
\hline Set 1 & Before Pre-processing & 61 \\
& Histogram Equalization & 78 \\
& CLAHE With image Entropy & 77 \\
\hline Set 2 & Before Pre-processing & 60 \\
& Histogram Equalization & 72 \\
& CLAHE With image Entropy & 77 \\
\hline
\end{tabular}

In phase two experimentation after pre-processing is performed and performance of pre-processing methods of face detection using three methods without preprocessing, using Histogram Equalization and using CLAHE with image entropy with respect to changing illumination and pose is analyzed. So for detection purposes extended Yale B dataset images are divided into three sets which are set 0 in , set 1 and set 2 based 
on various illumination conditions total 64 and pose of subjects in images. Set 0 contains frontal face images as well as 1 and 2 are with similar illumination condition with varying poses with 12 and 24 degree. As shown in table 2 face detection accuracy gets decreased with varying illumination and changing pose of subject.

As shown in table 3 average detection accuracy on the extended Yale B dataset is significantly improved using CLAHE with image entropy method than before pre-processing detection of faces. Experimental results show significant advantage against non-pre-processed detection of faces from the images in extended Yale B dataset. This is due to the effect of different illumination conditions initially facial parts are unclear and have a shadowing impact on them after the illumination

Table 3. Face detection performance on extended Yale B dataset

\begin{tabular}{|l|c|}
\hline $\begin{array}{l}\text { Illumination Pre- } \\
\text { processing Method }\end{array}$ & $\begin{array}{c}\text { Average } \\
\text { Detection } \\
\text { Accuracy (\%) }\end{array}$ \\
\hline Before Pre-processing & 62.66 \\
\hline Histogram Equalization & 80.33 \\
\hline CLAHE with Image Entropy & 81.66 \\
\hline
\end{tabular}

pre-processing these features like eyes and others are more clearly visible and thus more number of faces are successfully detected.

Face recognition is performed using PCA, LBP and Hybrid approach. Hybrid approach provides better results than the individual method as in this approach the integrated facial features of PCA with LBP are utilized. Calculating the global feature using PCA and getting the local or texture features of facial parts likes eyes and using them in training and testing of the faces. Face recognition performance is as shown in table 4. Recognition results on extended Yale B dataset using preliminary Histogram Equalization approach and hybrid approach as recognition method are listed where hybrid approach with CLAHE with image entropy for illumination method in this work has shown improved performance of $5.8 \%$.

CLAHE with clip limit 0.01 and $B S=8 \times 8$ is basic CLAHE. Table 5 shows details of evolution using basic CLAHE and another approach CLAHE with image entropy based illumination pre-processing. Input images are cropped frontal faces from both of the dataset. From the different recognition methods for evaluation, Hybrid approach of feature extraction and CLAHE with image entropy as illumination pre-processing provides higher performance as compared to other approaches.

Table 4. Face recognition performance on extended Yale B

\begin{tabular}{|l|c|c|c|}
\hline Dataset & $\begin{array}{c}\text { Recognition method } \\
\text { for evaluation }\end{array}$ & $\begin{array}{c}\text { Illumination } \\
\text { Pre-processing } \\
\text { Method }\end{array}$ & $\begin{array}{c}\text { Recognition } \\
\text { Rate (\%) }\end{array}$ \\
\hline $\begin{array}{l}\text { Extended } \\
\text { Yale B Dataset }\end{array}$ & Hybrid(PCA and LBP) & HE & 74.9 \\
\hline & Hybrid(PCA and LBP) & $\begin{array}{c}\text { CLAHE with } \\
\text { Image Entropy }\end{array}$ & 80.7 \\
\hline
\end{tabular}

Table 5. Face recognition Performance on Yale B \&t extended Yale B

\begin{tabular}{|l|c|c|c|}
\hline Dataset & $\begin{array}{c}\text { Recognition method } \\
\text { for evaluation }\end{array}$ & $\begin{array}{c}\text { Illumination } \\
\text { Pre-processing } \\
\text { Method }\end{array}$ & $\begin{array}{c}\text { Recognition } \\
\text { Rate (\%) }\end{array}$ \\
\hline \multirow{2}{*}{$\begin{array}{l}\text { Frontal faces of } \\
\text { combined dataset }\end{array}$} & PCA & Basic CLAHE & 79.2 \\
\hline \multirow{5}{*}{} & LBP & Basic CLAHE & 84.1 \\
\cline { 2 - 4 } & Hybrid(PCA and LBP) & Basic CLAHE & 85 \\
\cline { 2 - 4 } & PCA & CLAHE with Image Entropy & 85.7 \\
\cline { 2 - 4 } & LBP & CLAHE with Image Entropy & 86.3 \\
\cline { 2 - 4 } & Hybrid (PCA and LBP) & CLAHE with Image Entropy & 92 \\
\hline
\end{tabular}




\section{RESULTS ANALYSIS AND DISCUSSION}

In Histogram Equalization it is observed that, it over amplifies certain areas and increases the contrast of background noise, while decreasing the usable signal in some face images. Some of the failure cases of face detection are non- face like face image background or single eye on face are wrongly detected as faces. Further exceed amplification producing a lower performance in face recognition as compared to CLAHE pre-processing method. In basic CLAHE histogram is cut at some extent further HE is applied for improving local contrast of image. To avoid the amount of over implication, the peak of the histogram in every tile is truncated. In basic CLAHE here applied CL and BS parameters are fixed though illumination conditions are varying so noticeable visual improvement not able to produce. Whereas CLAHE with image entropy is adaptable to select CL value with the help of entropy of image in different imaging conditions. It is observed that BS has least impact on entropy value ultimately on quality of image.

In the analysis it shows that as CL increases with the step value the quality metric entropy parameter initially is increasing but after certain values it reaches saturation and remains constant throughout the scale. Thus selecting the saturated point CL value. This analysis of visibility along with the corresponding entropy of input images helps improve face detection and face recognition results. For most of the very low contrast images the observed average range of entropy value approximately with minimum and maximum as depicted in Fig 4. with varying illumination conditions. As dataset images are mostly dark and low contrast, which can be vary for other set of input images. As entropy provides the information about richness of content in the image, most of the lower range image entropy are observed for very dark and low contrast images whereas the range increase towards maximum value with better visibility and good contrast in the image. The important noting is that after the saturated point clip limit if it increases then thought the entropy remain in the same range but the over amplification and unbalance contrast effect is added in the image. So the clip limit selection is very decisional at the first saturated point of entropy.

Location of the human faces in the image can be found by using Haar features. A common universal property about all human faces is related to their facial features like the region of eyes is darker compared to its neighbor pixels and the region of nose is brighter as compared to eye region is benefited for local feature extraction. But in face detection phase some of the detection portion of the eye, upper head portion, and lower neck portion are falsely detected as faces. When in the datasets where their separation is not on the highest variance, then the use of the most important components of PCA will not work. One limitation of the basic LBP operator is that its small $3 \times 3$ neighborhood is unable to capture dominant features with large scale structures. Thus compared to individual methods PCA and LBP of feature extraction hybrid features of these techniques perform better using CLAHE with image entropy pre-processing approach for face recognition.

\section{CONCLUSION}

Illumination pre-processing is an effective approach for elimination of lighting variations before face detection and recognition. Representative illumination preprocessing approaches Histogram Equalization basic or fixed Contrast Limited Adaptive Histogram Equalization and Contrast Limited Adaptive Histogram Equalization with image entropy are analyzed briefly with respect to facial images. These approaches are evaluated for face recognition in various illumination conditions on Yale $\mathrm{B}$ and extended Yale B datasets.

With the use of CLAHE with image entropy method for illumination pre-processing significant improvement is observed in face detection and recognition for Yale B and extended Yale B dataset images. The face detection performance for illumination variation with pose variation image is low compared to the frontal images with same illumination condition. As the pose changes with the same illumination condition face detection performance decreases it is due to illumination impact on different facial parts. In face detection before any illumination pre-processing detection rate is $62.66 \%$ whereas after applying illumination pre-processing using CLAHE with image entropy, it is improved up to $81.66 \%$. Face recognition on extended Yale B using hybrid approach of Principal Component Analysis and Local Binary Pattern and CLAHE with entropy illumination preprocessing is tested and it is observed improvising recognition performance as compared to Histogram Equalization illumination preprocessing. Hybrid approach applied on cropped frontal faces.

These faces are pre-processed using CLAHE with image entropy. It has shown better recognition performance as $92 \%$. The results for illumination pre-processing are noted which eliminate the lighting variation before face detection and recognition. From the experimental analysis CLAHE with image entropy illumination pre-processing approach outperforms than Histogram Equalization and basic CLAHE illumination pre-processing techniques. It is observed that pre-processing on images has significant improvement in the performance of detection and recognition. Thus illumination pre-processing before face detection and feature extraction process for low contrast and dark images is a very essential step which provides improved performance. In this work the main focus is for improving visibility of low contrast images towards face recognition. Still for performance enhancement significantly in changing illumination condition with respect variation of poses, blending of various approaches under the Gray Level Transformation and other methods can be studied and planned for implementation as future work. 


\section{ACKNOWLEDGEMENTS}

We are very thankful to all the authors of literature used in this work. The referred techniques and concepts are really found useful and essential in the field of face recognition.

\section{REFERENCES}

A. S. Georghiades, P. N. Belhumeur, and D. J. Kriegman 2001, "From few to many: Illumination cone models for face recognition under variable lighting and pose”. IEEE Trans. Pattern Anal. Mach. Intell. 23(6): 643-660.

A. Thamizharasi and J. S. Jayasudha. 2016, “An Illumination Invariant Face Recognition by Enhanced Contrast Limited Adaptive Histogram Equalization”, ICTACT Journal on Image and Video Processing, 6(4):1258-1266

Anil K. Jain 2008, Fundamentals of Digital Image Processing, Pearson Education

B. Chanda,D. Dutta Majumder 2009, Digital Image Processing and Analysis, PHI Learning

B. Martinkauppi,M. Soriano, M. Pietikainen 2003, "Detection of Skin Color under Changing Illumination: A Comparative Study", Proceedings of the 12th International Conference on Image Analysis and Processing.

Byong Seok Min, Dong Kyun Lim, Seung Jong Kim and Joo Heung Lee 2013, “ A Novel Method of Determining Parameters of CLAHE Based on Image Entropy", International Journal of Software Engineering and Its Applications, 7(5):113-120.

C. Wang and Z. Ye 2005, "Brightness Preserving Histogram Equalization with Maximum Entropy: A Variation Perspective", IEEE Trans. on Consumer Electronics, 51(4):1326-1334.

Chris Solomon,Toby Breckon. 2010, Fundamentals of Digital Image Processing A Practical Approach with Examples in MATLAB,A John \& Sons Wiley ,Ltd, Publication

Di Huang,Caifeng Shan, Mohsen Ardabilian, Yunhong Wang and Liming Chen 2011, " Local Binary Patterns and Its Application to Facial Image Analysis: A Survey", IEEE Transactions on Systems, Man, And CyberneticsPart C: Applications And Reviews, 41(6): 765-781

G. F. C. Campos, S. M. Mastelini, G. J. Aguiar, R. G. Mantovani, L. F. de Melo and S. Barbon 2019, "Machine learning hyperparameter selection for contrast limited adaptive histogram Equalization”, EURASIP J. Image Video processing

H. F. Chen, P. N. Belhumeur, and D. W. Jacobs 2000," In search of illumination invariants", In Proc. IEEE Conf. Comput. Vis. Pattern Recognit. 254-261.

Hu Han, Shiguang Shan, Xilin Chen, Wen Gao. 2013," A comparative study on illumination pre-processing in face recognition”, Elsevier, Pattern Recognition 46: 1691-1699.

J. Ruiz-del-Solar, J. Quinteros 2008, "Illumination compensation and normalization in Eigen space-based face recognition: a comparative study of different preprocessing approaches”, Pattern Recognition Letters 29 : 1966-1979.

Jharna Majumdar, Santhosh Kumar K L 2014," Modified ClAHE: An Adaptive Algorithm for Contrast Enhancement of Aerial, Medical and Underwater Images", International Journal of Computer Engineering \&Technology 5(11): 32-47.

Jiwen Lu, Venice Erin Liong, Xiuzhuang Zhouand Jie Zhou 2015,"Learning Compact Binary Face Descriptor for Face Recognition", IEEE Transactions on Pattern Analysis and Machine Intelligence, 37: 2041-2055.

K.C. Lee and J. Ho and D. Kriegman 2005, "Acquiring linear Subspaces for face recognition under variable lighting" IEEE Trans. Pattern Anal. Mach. Intelligence,27(5): 684698

Karl Zuiderveld.1994,"Contrast Limited Adaptive Histogram Equalization", Academic Press, Inc. 474484.

Kim Y.-T. 1997,"Contrast enhancement using brightness preserving Bi-Histogram equalization", IEEE Trans. Consumer Electronics, 43(1): 1-8.

Leyuan Liu, Nong Sang, Saiyong Yang and Rui Huang 2011, " Real-Time Skin Color Detection under Rapidly Changing Illumination Conditions", IEEE Transactions on Consumer Electronics 57(3):1295-1302.

M. Turk and A. Pentland. 1991," Eigenfaces for Recognition”. Journal of Cognitive Neuroscience, hard copy 3(1):71-86,

M.Anji Reddy,Y.Hari Shankar 2009, Textbook of Digital Image Processing, BSPublication

Nicholas SiaPikKong, Haidi Ibrahim and Seng Chun Hoo 2013, "A Literature on Histogram Equalization and Its Variation for Digital Image Enhancement" In International Journal of Innovation, Management and Technology, 4: 386-389.

Paul Viola, Michael J. Jones 2004, “ Robust Real Time Face Detection”, Int. Journal of Computer Vision ,57(2): 137-154.

Ping-Han Lee, Szu-Wei Wu, and Yi-Ping Hung 2012, "Illumination Compensation Using Oriented Local Histogram Equalization and Its Application to Face Recognition”, IEEE Transactions on Image Processing 21(9): 4280-4289.

R M Kagalkar, Nagaraj H.N, S V Gumaste 2015, “A Novel Technical Approach for Implementing Static Hand Gesture Recognition”. International Journal of Advanced Research in Computer and Communication Engineering, 4(7): 156-160.

R. Gray. 1990, Entropy and Information Theory Springer-Verlag

R. P.Dahake, M.U. Kharat 2018, “ Face Detection and Processing: a Survey”, International Journal of Engineering \&t Technology 7,4.19: 1066-1071.

Rafael C.Gonzalez, Richard E. Woods 2009, Digital Image Processing, Pearson Education 
Ranjan Parekh 2006, Principals of Multimedia, MATLAB Examples Second Edition Mc Graw Hill

S. Pizer, R. Johnston, J. Ericksen, B. Yankaskas, and K. Muller 1990, "Contrast-limited adaptive histogram equalization: Speed and effectiveness", In Proc. 1st Conf. Visual. Biomed. Comput. 337-345.

S. Shan, W. Gao, B. Cao, D. Zhao 2003, "Illumination normalization for robust face recognition against varying lighting conditions", In Proceedings of the ICCV Workshop on Analysis and Modeling of Faces and Gestures.157-164.

S.Anila \& Dr.N.Devarajan 2012, "Pre-processing technique for face recognition applications under varying illumination conditions", Global Journal of Computer Science and Technology Graphics \& Vision 12,11 Ver 1.0: 13-18

S.M. Pizer, E.P. Amburn, J.D. Austin, R. Cromartie, A. Geselowitz, T. Greer, B.H. Romeny, J.B. Zimmerman, K. Zuiderveld 1987, “Adaptive Histogram Equalization and its Variations",Computer Vision, Graphic, and Image Processing 39: 355-368.

W. Chen, M.J. Er, S. Wu. 2006, “ Illumination compensation and normalization for robust face recognition using discrete cosine transform in logarithm domain” IEEE Transactions on Systems, Man, and Cybernetics Part B: Cybernetics 36:458-466.

W. Zhao, R. Chellappa, A. Rosenfeld, and P. Phillips 2003, "Face recognition: A literature survey", ACM Computing Surveys 399-458.

X. Tan, B. Triggs 2010, "Enhanced local texture feature sets for face recognition under difficult lighting conditions”, IEEE Transactions on Image Processing, 19 (6): 1635 - 1650 .

X. Xie, W.-S. Zheng, J. Lai, and P. Yuen 2008, “ Face Illumination Normalization on Large and Small Scale features", In Proc. IEEE Conf. Comput.Vis. Pattern Recognit. 1-8.

X. Zou, J. Kittler, K. Messer 2007, "Illumination invariant face recognition: a survey", In Proceedings of the Biometrics: Theory, Applications, and Systems 1-8.

Y. Adini, Y. Moses, and S. Ullman 1997, "Face recognition: the problem of compensating for changes in illumination direction", IEEE Transactions on Pattern Analysis and Machine Intelligence 19: 721-732. 